\title{
Valutazione della competenza digitale: che cosa fare per la scuola primaria*
}

\author{
Antonio Calvani - Laura Menichetti \\ Università degli Studi di Firenze, Dipartimento di Scienze della Formazione \\ e Psicologia \\ doi: 10.7358/ecps-2014-010-meni \\ antonio.calvani@unifi.it \\ laura.menichetti@unifi.it
}

\section{ASSESSMENT OF DIGITAL COMPETENCE: A TOOL FOR PRIMARY SCHOOLS}

\section{Abstract}

Digital competence is now recognized as one of the most important educational goals to be pursued in the policies of the new millennium. Research is proposing numerous considerations to better define the nature of this competence and it generally stresses the need to develop theoretical models in which, together with strictly technical knowledge and skills, cognitive abilities and notions of ethics and behavior are also present. This theoretical reflection includes the problem of providing schools with tools to facilitate and assess progressive advances at various levels of age. In the European framework and within the Digital Competence Assessment (DCA), a program which also covers other school levels, the present contribution shows a questionnaire used in order to assess digital competence in primary schools, which is useful to identify areas on which to conduct immediate actions. The questionnaire, together with its accompanying instructions, is made available to schools under the Creative Commons license.

Keywords: Assessment, Digital competence, Model, Primary school, Questionnaire.

* Il presente contributo è esito di un lavoro ideato collaborativamente; la sua compilazione può essere attribuita per i parr. 3, 4, 7, 8 ad A. Calvani e per i parr. 1, 2, 5, 6 a L. Menichetti. 


\section{LA COMPETENZA DIGITALE}

La nozione di competenza digitale affonda le sue radici nell'avvento stesso del computer e nel tradizionale concetto di alfabetizzazione informatica che ha accompagnato l'introduzione delle nuove tecnologie nella scuola e nel lavoro a partire dagli anni Ottanta. A mano a mano che le tecnologie acquisivano nuove connotazioni ed implicazioni cognitive e sociali anche la riflessione è venuta diversificandosi. Accanto ad approcci tecnologico-funzionali ancorati a conoscenze di tipo informatico (Bruce \& Peyton, 1999; Davies, Szabo, \& Montgomerie, 2002; Swan et al., 2002), se ne sono affermati altri che hanno messo al centro l'informazione (accesso, elaborazione, trasferimento, conservazione) sulla scia di orientamenti biblioteconomici (ALA, 1989; ACRL, 2000) oppure la capacità di comprendere i principi e le strategie necessarie per sviluppare soluzioni tecnologiche e gli effetti sociali ed etici che ne derivano (es. NAEP, 2010).

Indipendentemente dalle diversità, nel corso dei decenni si è affermato un progressivo spostamento di accento da una concezione basata su una pura conoscenza tecnica, verso un approccio integrato con altre dimensioni come quella critico-cognitiva (es. il saper trattare informazioni e dati) e quella della responsabilità civica e sociale (es. il saper rispettare gli altri e tutelare la propria sicurezza nella rete), che hanno trovato una autorevole sintesi nella Raccomandazione Europea 962 del 2006, relativa alle competenze chiave per l'apprendimento permanente (EU, 2006) ${ }^{1}$.

Negli ultimi anni ulteriori ricerche e progetti hanno perfezionato questo riferimento, cercando anche di favorirne le implementazioni didattiche. Un contributo teorico rilevante è derivato dal recente progetto $D i g C o m p^{2}$, articolatosi in tre fasi principali: la definizione di un modello di competenza digitale (Ala-Mutka, 2011), la selezione dei modelli di competenza digitale più significativi a livello europeo per trarne una definizione operativa di sintesi (Ferrari, 2012), una consultazione di esperti per rispondere alla domanda su chi possa essere definito digitalmente competente. Il progetto evidenzia la necessità di identificare un quadro di conoscenze stabili (capacità d'uso di strumenti e applicazioni digitali, depurate da tecnicismi superficiali) che deve entrare in rela-

1 Nella Raccomandazione Europea del 2006 la competenza digitale è definita come il «saper utilizzare con dimestichezza e spirito critico le tecnologie della società dell'informazione per il lavoro, il tempo libero e la comunicazione» e si sottolinea l'importanza della tecnologie «a sostegno del pensiero critico, della creatività e dell'innovazione». La competenza digitale viene annoverata tra quelle competenze «di cui tutti hanno bisogno per la realizzazione e lo sviluppo personali, la cittadinanza attiva, l'inclusione sociale e l'occupazione» (EU, 2006).

2 DigComp: «Digital Competence; identification and European-wide validation of its key components for all levels of learners». 
zione con competenze strategiche (gestione dell'informazione, collaborazione, comunicazione e condivisione, creazione di contenuti e di conoscenza, etica e responsabilità, valutazione e problem solving). La competenza digitale consiste nell'attivazione di queste relazioni bidirezionali tra conoscenze tecniche e competenze strategiche (Calvani \& Menichetti, 2013; Olimpo, 2013).

\section{Il MODELLO DCA}

Dietro l'impulso della Raccomandazione Europea (EU, 2006) è nato anche il modello Digital Competence Assessment (DCA) ${ }^{3}$, che accompagna l'elaborazione teorica con strumenti di valutazione pratici impiegabili nei diversi livelli scolastici e differenziati per complessità (Calvani, Fini, \& Ranieri, 2009a, 2009c e 2010; Calvani et al., 2012; Calvani \& Menichetti, 2013; Didoni et al., 2013).

La competenza digitale, secondo la definizione adottata nell'ambito del progetto DCA, consiste nel saper esplorare ed affrontare in modo flessibile situazioni tecnologiche nuove, nel saper analizzare selezionare e valutare criticamente dati e informazioni, nel sapersi avvalere del potenziale delle tecnologie per la rappresentazione e la soluzione di problemi e per la costruzione condivisa e collaborativa della conoscenza, mantenendo la consapevolezza della responsabilità personali, del confine tra sé e gli altri e del rispetto dei diritti/doveri reciproci (Calvani, Fini \& Ranieri, 2009b).

Il modello che deriva dalla definizione (Figura 1) si caratterizza per una integrazione tra conoscenze di natura tecnologica con altre di natura eticorelazionale e con abilità cognitive, in parte trasversali ad altre literacy di base. Tale modello accoglie alcune suggestioni pedagogiche che sono emerse via via che le tecnologie si sono presentate nella scuola nel corso degli ultimi trent'anni: Esplorazione, Simulazione, Inquiry, Collaborazione, Partecipazione. Esse possono diventare altrettante piste secondo le quali valutare la competenza digitale (Calvani, Fini \& Ranieri, 2010).

Il modello DCA è stato preso in considerazione anche dal progetto DigComp (2010-2012) ed è possibile ravvisare rilevanti punti di convergenza con la sintesi degli altri framework proposti a livello europeo, in particolare per quanto attiene la connotazione non puramente tecnica, ma in stretto rapporto con dimensioni critico-cognitive ed etico-sociali (Eshet-Alkalai, 2004; Ala-Mutka, 2011; Ferrari, 2012; Janssen \& Stoyanov, 2012) (Figura 2).

3 Progetto di Ricerca di Interesse Nazionale (PRIN MIUR DM 582/2006 del 24 marzo 2006), Internet e scuola: problematiche di accessibilità, politica delle uguaglianze e gestione dell'informazione, coordinato da Antonio Calvani, Università degli Studi di Firenze. 


\section{ESPLORAZIONE}

esplorare nuovi contesti tecnologici ed affrontare in modo flessibile situazioni tecnologiche nuove

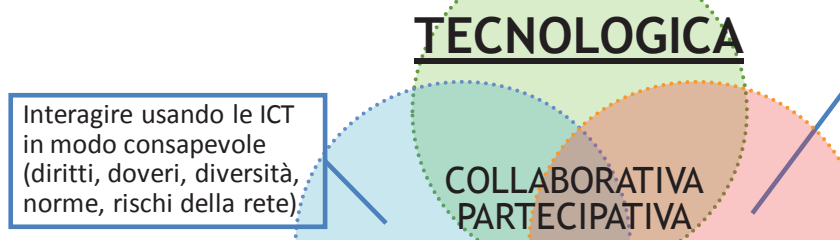

ETICA

COGNITIVA
Analizzare l'informazione, selezionare, valutare. Avvalersi delle tecnologie per la rappresentazione e la

soluzione di problemi

\section{SIMULAZIONE}

\section{INQUIRY}

ETro

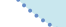

Comprendere il potenziale delle tecnologie per la costruzione collaborativa di conoscenza (sul piano emozionale/motivazionale o del senso civico)

\section{COLLABORAZIONE PARTECIPAZIONE}

Figura 1. - Modello DCA (Calvani, Fini, \& Ranieri, 2010).

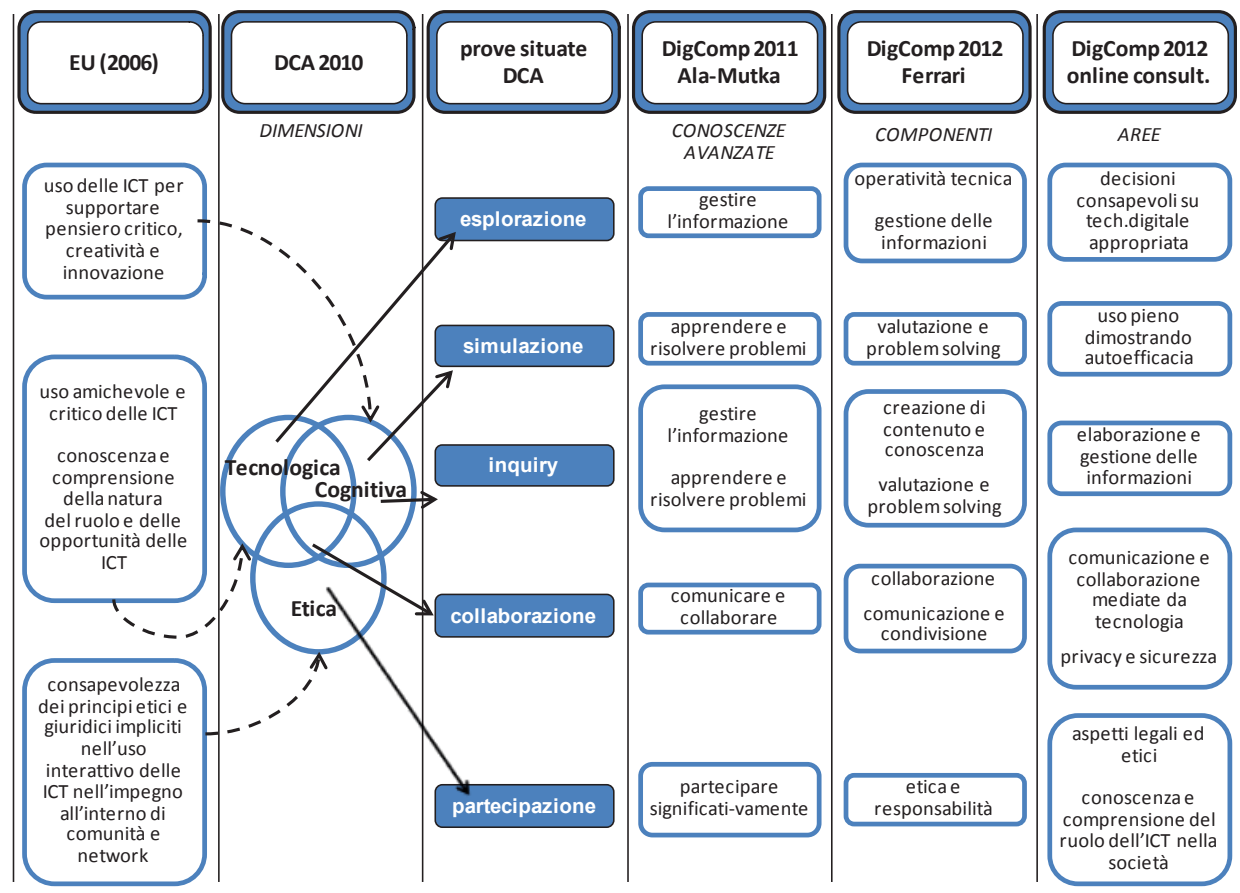

Figura 2. - Comparazione tra il modello DCA e altri modelli oggetto di ricerca internazionale (Calvani \& Menichetti, 2013). 
In Figura 2 le prime due colonne mostrano il raccordo tra il modello DCA e la Raccomandazione Europea del 2006; la terza rimanda alle cinque piste applicative del modello, rese oggetto di prova di valutazione; la parte destra sintetizza l'esito dei principali documenti redatti nel corso del progetto DigComp, sempre con allineamento delle similarità (Calvani \& Menichetti, 2013).

\section{LE PROVE IDCA}

La sfida posta a livello europeo dal riconoscimento della competenza digitale come una delle competenze chiave di cittadinanza (EU, 2006), accolta anche dalle normative italiane che sollecitano la certificazione delle competenze nell'ambito dei vari ordini scolastici (DPR 122/2009; DM 9/2010) esige ormai di passare alla implementazione di percorsi didattici corredati da adeguati sistemi di valutazione.

La complessità insita nella nozione di competenza indurrebbe ad accogliere il suggerimento di impiegare compiti autentici (Wiggings, 1991; Silva, 2008) tenendo tuttavia conto della concreta gestibilità didattica e dell'affidabilità della valutazione. La strada più ragionevole appare quella di perseguire un approccio che affianchi il testing tradizionale con prove più complesse. Per fare ciò ci siamo richiamati ad un orientamento in uso in ambiti aziendali (Cognitive Task Analysis, CTA; Crandall et al., 2006) che richiede di partire inizialmente dalla rappresentazione di situazioni realistiche rappresentative della competenza, attraverso informazioni acquisite da esperti (challenging scenario) procedendo poi ad un'opera di semplificazione (short scenario, traducibili in esercizi situati, di una durata dell'ordine di un'ora), isolando infine conoscenze e skill cognitive essenziali valutabili attraverso un testing tradizionale (iDCA, dove «i» significa instant). Quest'ultime prove (iDCA), di cui presentiamo un esempio, sono dunque parte di un complesso più articolato. Le conoscenze selezionate riguardano la parte tecnologica ed etica, le abilità quella cognitiva. Le conoscenze tecnologiche sono relative a situazioni di problem solving tipiche (trouble shooting) o alla rappresentazione che il soggetto ha del computer e della rete, delle loro potenzialità e criticità. Le conoscenze etiche, in linea con l'attenzione crescente su questi aspetti accentuatasi dopo l'avvento del web 2.0, riguardano essenzialmente la sicurezza, il rispetto dei diritti degli altri e la consapevolezza del divario digitale. La dimensione cognitiva concerne abilità cognitive di livello alto, come saper identificare $\mathrm{i}$ termini essenziali di un testo, saper organizzare gerarchicamente dei dati in classi, saper inferire i dati da una tabella, oggetto anche delle misurazioni internazionali più diffuse (TIMSS, PIRLS, PISA). 
Prove iDCA sono state sviluppate fin dal 2009/10 (Calvani, Fini, \& Ranieri, 2010). Nella versione per il biennio della scuola secondaria di secondo grado (età 15-16 anni) sono state oggetto prima di una fase di item analysis condotta in Italia e in Cina (Li \& Ranieri, 2010) e poi di una sperimentazione a livello nazionale che ha visto coinvolti 1.117 studenti appartenenti a 37 scuole diverse.

Il presente contributo presenta la versione iDCA per la scuola primaria e ne analizza i risultati ${ }^{4}$.

\section{LA COMPETENZA DIGITALE NELLA SCUOLA PRIMARIA}

Una criticità in cui spesso ci si imbatte con insegnanti che intendono sviluppare la competenza digitale è la loro difficoltà nell'identificare obiettivi significativi, circoscrivibili e verificabili. La maggior parte di essi pensa che la strada giusta consista nell'accompagnare quanto più possibile le pratiche di studio nelle diverse discipline con l'uso del computer: così ad esempio il semplice uso della Lim, dei tablet, oppure la progettazione multimediale sembrerebbero le modalità più valide per conseguire competenza digitale unitariamente all'acquisizione dei contenuti. La ricerca Evidence Based (Hattie, 2009) ci mostra che gli apprendimenti disciplinari non migliorano con le tecnologie mentre per ciò che riguarda lo sviluppo di competenza digitale, laddove questa non sia stata adeguatamente identificata sul piano concettuale, si genera dispersione di tempo in attività di bassa rilevanza educativa in rapporto allo scopo.

È importante che gli educatori comprendano che la competenza digitale non si identifica con le maestrie tecniche che solitamente caratterizzano le pratiche tecnologiche dei cosiddetti «nativi digitali» (Prensky, 2001), quali tagliare, incollare, mettere video su YouTube, e attività simili: non si è competenti digitali se non si ha un'idea realistica dei meccanismi che stanno dietro all'esecuzione di un software o di un collegamento in rete, se non si sa leggere e selezionare criticamente informazioni testuali, se non si sanno costruire gerarchie e tabelle, se non si comprende la distinzione tra reale e virtuale e come il mondo della rete sia pieno di insidie e richieda forte autocontrollo e responsabilità. Anche le Indicazioni nazionali per il curricolo della scuola dell'infanzia e del primo ciclo d'istruzione (DM 254 del 16 novembre 2012)

4 Allo sviluppo delle versioni hanno contribuito nel tempo Patrizia Appari, Laura Menichetti, Stefania Carioli, Claudia Calvani, Letizia Balbi, Silvia Giovacchini. La versione attuale è la 4. 
assumono come orizzonte di riferimento il pieno sviluppo delle competenze chiave per il lifelong learning definite dall'Unione Europea (EU, 2006) tra cui si annovera la competenza digitale. Secondo le aspettative delle Indicazioni il ragazzo che termina la secondaria di I grado "ha buone competenze digitali, usa con consapevolezza le tecnologie della comunicazione per ricercare e analizzare dati ed informazioni, per distinguere informazioni attendibili da quelle che necessitano di approfondimento, di controllo e di verifica e per interagire con soggetti diversi nel mondo», come si legge nel Profilo delle competenze al termine del primo ciclo di istruzione. Si afferma anche che "oltre alla padronanza degli strumenti, spesso acquisita al di fuori dell'ambiente scolastico, si sviluppi un atteggiamento critico e una maggiore consapevolezza rispetto agli effetti sociali e culturali della loro diffusione, alle conseguenze relazionali e psicologiche dei possibili modi d'impiego, alle ricadute di tipo ambientale o sanitario, compito educativo cruciale che andrà condiviso tra le diverse discipline».

\section{LA PROVA IDCA PER LA SCUOLA PRIMARIA}

A partire dal 2012, in linea con la filosofia generale del progetto e con le prove già realizzate per i livelli scolastici superiori, abbiamo avviato un programma per predisporre una prova iDCA per la scuola primaria, capace in un tempo molto contenuto di offrire un quadro sufficientemente rappresentativo del posizionamento del singolo soggetto e della classe, in rapporto ad un benchmark rappresentato da un universo statistico di riferimento, per quanto riguarda tutte le dimensioni in cui si articola il concetto di competenza digitale. Lo scopo è diagnostico e formativo: una volta identificati i settori di particolare criticità per i bambini di una determinata classe, sarà possibile fornire indicazioni per colmare il gap relativo.

La versione completa del test, originato da un numero più ampio di item e passato attraverso una preliminare item analysis, è costituita da 48 item, che coprono le dimensioni tecnologica, cognitiva ed etica, frazionabile in due metà equivalenti da 24 item ciascuna. Il test si rivolge a bambini al termine della quarta della scuola primaria o all'inizio della quinta.

All'interno di ogni dimensione sono individuati ambiti specifici, a ciascuno dei quali sono dedicati complessivamente quattro item, collocati a due a due in sezioni diverse del questionario. Tali ambiti sono rappresentati in Figura 3. 


\section{iDCA per la scuola primaria}

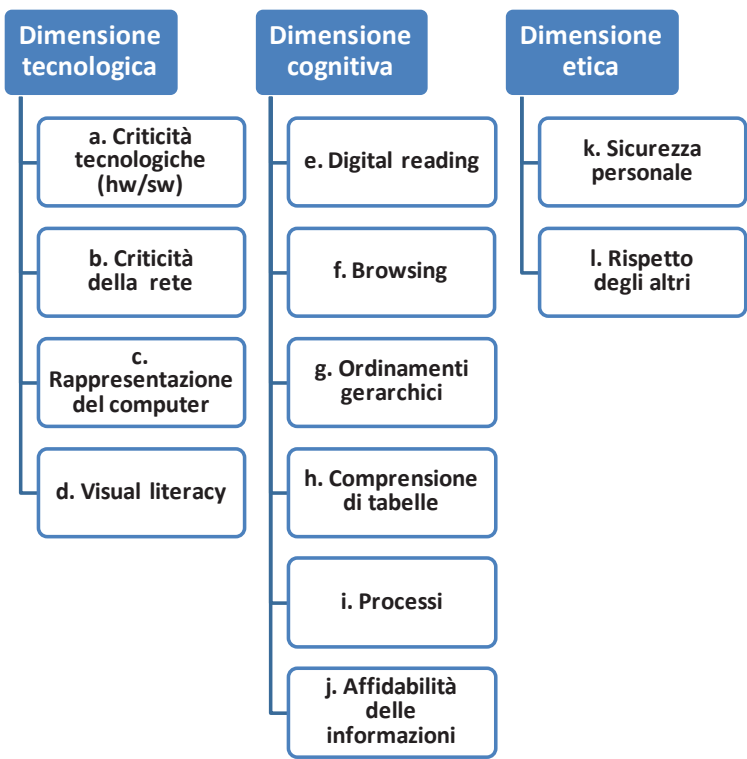

Figura 3. - Syllabus del test.

La prova è a scelta multipla. Ogni item consente una, due, o tre risposte corrette. Il punteggio massimo relativo ad ogni item è comunque sempre pari a 1 , eventualmente attribuito in maniera frazionata in proporzione alle risposte corrette fornite $(0,5$ per ciascuna delle due risposte previste, oppure 0,33 per ciascuna delle tre risposte previste).

L'applicazione richiede un preliminare controllo lessicale e, se necessario, un breve intervento di integrazione informativa prima della somministrazione. Al termine dell'applicazione si forniscono immediatamente un feedback sulle risposte e informazioni sui punti risultati più critici ${ }^{5}$.

Un esempio di item è riportato in Figura 4.

5 La versione più recente del test, la 4 (iDCA $\left.\mathrm{ill.v4}_{4}\right)$, è disponibile sotto licenza Creative Commons (CC BY-NC-ND 3.0) sul sito www.educadigitale.it. Sullo stesso sito è reperibile anche una corrispondente versione facilitata, da applicare con soggetti che presentano lievi disabilità sul fronte cognitivo o carenze sul piano linguistico, oppure nella classe terza della scuola primaria. La realizzazione di kit di intervento didattico, funzionali al raggiungimento di specifici obiettivi, il cui effettivo conseguimento può essere verificato alla fine dell'intervento, è oggetto di elaborazione. 


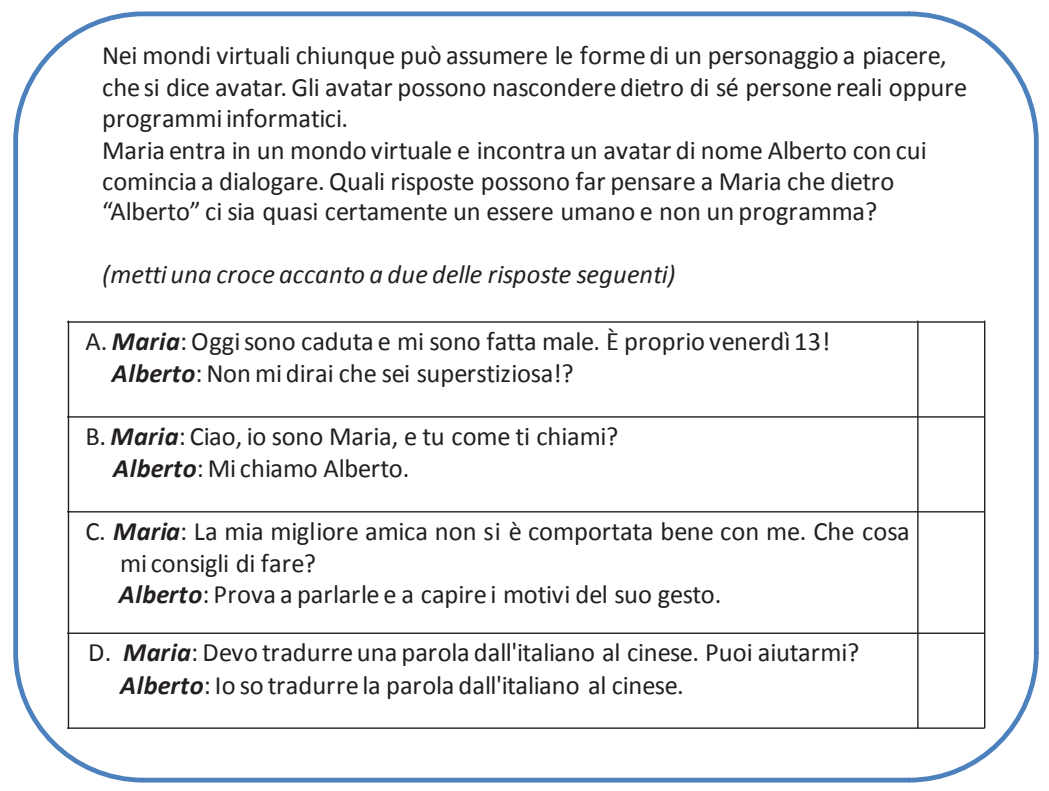

Figura 4. - Esempio di item. Le risposte corrette sono A e C.

\section{SPERIMENTAZIONE E ANALISI DEI DATI}

La prova è stata applicata nella versione 3 in 18 classi della scuola primaria, ubicate in Toscana, per un totale di 273 bambini. Le classi si trovano al $28 \%$ in area urbana (anche se non in capoluoghi di provincia) e per il $72 \%$ in aree più periferiche: non abbiamo però rilevato differenze statisticamente significative tra i due gruppi. La selezione dei soggetti non è stata di natura randomica ma basata sulla disponibilità degli insegnanti; in ogni caso hanno partecipato classi intere e non gruppi selezionati di allievi.

L'applicazione ci ha consentito di apportare dei miglioramenti allo strumento ${ }^{6}$ e di avanzare qualche considerazione sulle difficoltà manifestate dai bambini.

6 I dati qui elaborati fanno riferimento alla versione 3. Il questionario nella versione 2 aveva già usufruito di una precedente item analysis. Sul sito www.educadigitale.it viene fornita la versione 4 nella quale alcuni item sono stati ulteriormente ritoccati per tenere conto meglio degli indici di facilità. 


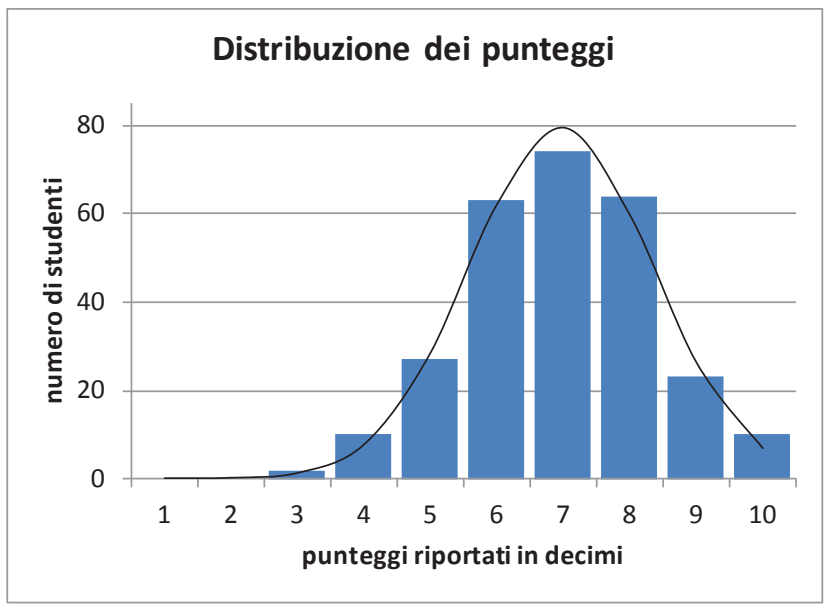

\begin{tabular}{|l|r|}
\hline numero di item & 48 \\
\hline numero di casi & 273 \\
\hline media & 6,46 \\
\hline mediana & 6,53 \\
\hline dev. standard & 1,37 \\
\hline varianza & 1,87 \\
\hline curtosi & $-0,10$ \\
\hline minimo & 2,19 \\
\hline massimo & 9,69 \\
\hline
\end{tabular}

Figura 5. - Distribuzione dei punteggi riportati dagli studenti.

Nella Figura 5 è rappresentata la curva dei punteggi ottenuti, che come si vede è approssimabile ad una gaussiana, con un leggero spostamento dei punteggi verso i valori più positivi. Tale caratteristica garantisce una complessiva adeguatezza del test all'età considerata e il fatto che il campione possa essere preso a riferimento come benchmark in attesa di sperimentazioni a livello nazionale.

Data la natura composita della competenza digitale, attivabile da esperienze e riflessioni afferenti ad ambiti diversi così come previsto dal modello, non è lecito aspettarsi valori di correlazione alti tra gli item come solitamente si ottengono in test che riguardano attitudini o abilità più omogenee e coese. In ogni caso l'indice alpha di Cronbach per l'intero questionario da 48 domande attesta una sostanziale consistenza interna dello strumento $(\alpha=0,82)$.

L'applicazione del test completo (48 item) consente maggiore affidabilità, però è possibile anche applicare una sola delle due metà mantenendo una sostanziale rappresentatività dei risultati. Pur non avendo seguito esattamente un processo di split-half, abbiamo garantito una corrispondenza tra le due sezioni in termini di difficoltà media e di deviazione standard (nella Figura 6 è possibile confrontare ogni riga «ingresso» con la corrispondente riga «uscita»). L'indice di correlazione di Pearson tra le due prove è pari a 0,71 .

È possibile anche proporre le due applicazioni a distanza di tempo, come prove una di ingresso e l'altra di uscita rispetto ad un intervento didattico. 


\begin{tabular}{|l|c|c|c|c|}
\hline & TOTALE & $\begin{array}{c}\text { DIMENSIONE } \\
\text { TECNOLOGICA }\end{array}$ & $\begin{array}{c}\text { DIMENSIONE } \\
\text { COGNITIVA }\end{array}$ & $\begin{array}{c}\text { DIMENSIONE } \\
\text { EIICA }\end{array}$ \\
\hline MEDIA IN+OUT /10 & 6,46 & & & \\
\hline MEDIA INGRESSO /10 & 6,42 & 7,02 & 5,82 & 7,01 \\
\hline MEDIA USCITA /10 & 6,51 & 7,14 & 5,89 & 7,09 \\
\hline & & & & \\
\hline DEV. STANDARD IN+OUT /10 & 1,37 & & & \\
\hline DEV. STANDARD INGRESSO /10 & 1,43 & 1,54 & 1,98 & 2,74 \\
\hline DEV. STANDARDS USCITA/10 & 1,51 & 1,66 & 1,81 & 2,67 \\
\hline
\end{tabular}

Figura 6. - Le due metà del test: confronto di media e deviazione standard (i punteggi sono riportati in decimi).

La Figura 6, oltre a suffragare la possibilità di usare il test in forma splittata, offre informazioni circa il risultato conseguito dagli studenti nelle tre diverse dimensioni: la media dei risultati nella dimensione tecnologica e in quella etica supera i 7 punti (su 10), mentre nella dimensione cognitiva risulta al di sotto dei 6 punti $(5,82$ e 5,89 su 10). Questo risultato è ricorrente ed è stato registrato anche in applicazioni ulteriori rispetto a quella esaminata nel presente contributo.

La Figura 7 sintetizza i risultati ottenuti dall'intero questionario ordinandoli dal più facile al più difficile; le barre più chiare corrispondono agli item afferenti alla dimensione tecnologica, quelle di colore medio alla dimensione etica e quelle più scure alla dimensione cognitiva. Le righe orizzontali di maggior spessore separano i quattro quartili. Per ogni item si riporta il numero progressivo, la voce di riferimento nel Syllabus, l'argomento trattato, l'Indice di Facilità (IF) ${ }^{7}$, la Deviazione Standard (DS) e l'Indice di Discriminazione (ID) ${ }^{8}$.

7 L'Indice di Facilità è qui definito come $\mathrm{IF}=\mathrm{X}_{\text {medio }} / \mathrm{X}_{\max }$ dove $\mathrm{X}_{\text {medio }}$ è il punteggio

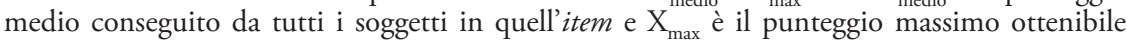
dell'item stesso (nel caso specifico 1). Secondo McAlpine (2002) item troppo facili (IF > 0,85) o troppo difficili $(\mathrm{IF}<0,1)$ devono essere eliminati perché non consentono un'efficace discriminazione. Nel caso specifico si rileva che soltanto 2 item hanno superato la soglia consigliata, risultando facili, mentre nessun item è risultato così difficile da scendere sotto al valore 0,1 . I due item più facili sono stati modificati nella versione 4 riportata nel sito.

8 L'Indice di Discriminazione è qui definito come $I D=\left(E_{M}-E_{P}\right) / n_{M}$ dove $E_{M}$ è la somma dei risultati conseguiti nel terzile superiore (i risultati migliori), $\mathrm{E}_{\mathrm{P}}$ è la somma dei risultati conseguiti nel terzile inferiore (i risultati peggiori), $\mathrm{n}_{\mathrm{M}}$ è il numero corrispondente ad $1 / 3$ dei soggetti partecipanti (in questa sperimentazione $\mathrm{n}_{M}=91$ ). Nel caso specifico non si rilevano indici di discriminazione negativi. 


\begin{tabular}{|c|c|c|c|c|c|c|c|c|c|c|}
\hline ITEM & SYLLABUS & ARGOMENTO & IF & DS & ID & 0,2 & 0,4 & 0,6 & 0,8 & 1,0 \\
\hline 26 & crit. tech. hw\&sw & malfunzionamento software & 0,943 & 0,170 & 0,225 & & & & & \\
\hline 36 & browsing & ricerca di informazioni in rete & 0,879 & 0,326 & 0,187 & & & & & \\
\hline 2 & crit. tech. hw\&sw & malfunzionamento hardware & 0,850 & 0,266 & 0,176 & & & & & \\
\hline 31 & visual literacy & riconoscimento di icone & 0,847 & 0,279 & 0,194 & & & & & \\
\hline 47 & rispetto degli altri & diffusione in rete di foto di amici & 0,828 & 0,378 & 0,473 & & & & & \\
\hline 23 & rispetto degli altri & diffusione in rete di foto di amici & 0,824 & 0,381 & 0,209 & & & & & \\
\hline 3 & criticità della rete & malfunzionamento nella visualizzazione di pagine web & 0,819 & 0,255 & 0,198 & & & & & \\
\hline 7 & visual literacy & riconoscimento di icone & 0,813 & 0,197 & 0,114 & & & & & \\
\hline 1 & crit. tech. hw\&sw & malfunzionamento software & 0,808 & 0,258 & 0,165 & & & & & \\
\hline 9 & digital reading & comprensione di un testo (in un blog) & 0,806 & 0,298 & 0,187 & & & & & \\
\hline 24 & rispetto degli altri & diffusione in rete di video di amici & 0,799 & 0,401 & 0,319 & & & & & \\
\hline 43 & affidabilità info. & valutazione di affidabilità di un'informazione & 0,744 & 0,437 & 0,352 & & & & & \\
\hline 46 & sicurezza personale & dare fiducia a interlocutori occasionali in rete & 0,744 & 0,321 & 0,286 & & & & & \\
\hline 48 & rispetto degli altri & diffusione in rete di video di amici & 0,729 & 0,445 & 0,286 & & & & & \\
\hline 19 & affidabilità info. & valutazione di affidabilità di un'informazione & 0,718 & 0,450 & 0,396 & & & & & \\
\hline 20 & affidabilità info. & valutazione di affidabilità di fonti reperite in rete & 0,714 & 0,452 & 0,385 & & & & & \\
\hline 25 & crit. tech. hw\&sw & malfunzionamento hardware & 0,712 & 0,295 & 0,115 & & & & & \\
\hline 21 & sicurezza personale & dare fiducia a interlocutori occasionali in rete & 0,707 & 0,455 & 0,297 & & & & & \\
\hline 27 & criticità della rete & malfunzionamento nella visualizzazione di pagine web & 0,707 & 0,268 & 0,126 & & & & & \\
\hline 11 & browsing & ricerca di informazioni in rete & 0,703 & 0,457 & 0,253 & & & & & \\
\hline 32 & visual literacy & interpretazione di interfacce (barra di menu) & 0,703 & 0,457 & 0,330 & & & & & \\
\hline 30 & rappres. computer & interazioni attraverso pc: con persone e con sistemi & 0,690 & 0,381 & 0,429 & & & & & \\
\hline 44 & affidabilità info. & valutazione di affidabilità di fonti reperite in rete & 0,685 & 0,465 & 0,187 & & & & & \\
\hline 12 & browsing & ricerca di informazioni in rete & 0,670 & 0,470 & 0,187 & & & & & \\
\hline 35 & browsing & ricerca di informazioni in rete & 0,670 & 0,470 & 0,319 & & & & & \\
\hline 6 & rappres. computer & interazioni attraverso pc: con persone e con sistemi & 0,668 & 0,409 & 0,385 & & & & & \\
\hline 4 & criticità della rete & messaggistica conseguente all'invio di e-mail & 0,612 & 0,487 & 0,209 & & & & & \\
\hline 33 & digital reading & comprensione di un testo (in un blog) & 0,612 & 0,328 & 0,280 & & & & & \\
\hline 40 & comprens. tabella & descrizione di un fenomeno attraverso una tabella & 0,604 & 0,489 & 0,308 & & & & & \\
\hline 18 & processi & esecuzione di un percorso guidato & 0,590 & 0,492 & 0,374 & & & & & \\
\hline 37 & ordinam. gerarchici & i organizzazione gerarchica delle pagine di un sito & 0,590 & 0,492 & 0,396 & & & & & \\
\hline 29 & rappres. computer & riconoscimento di avatar & 0,588 & 0,344 & 0,423 & & & & & \\
\hline 16 & comprens. tabella & descrizione di un fenomeno attraverso una tabella & 0,575 & 0,494 & 0,319 & & & & & \\
\hline 5 & rappres. computer & riconoscimento di avatar & 0,557 & 0,342 & 0,385 & & & & & \\
\hline 17 & processi & lettura di un diagramma di flusso & 0,549 & 0,498 & 0,429 & & & & & \\
\hline 45 & sicurezza personale & dare fiducia a interlocutori occasionali in rete & 0,535 & 0,499 & 0,341 & & & & & \\
\hline 41 & processi & lettura di un diagramma di flusso & 0,524 & 0,499 & 0,473 & & & & & \\
\hline 28 & criticità della rete & prerequisiti per inviare una mail & 0,516 & 0,500 & 0,286 & & & & & \\
\hline 38 & ordinam. gerarchici & i organizzazione gerarchica di informazioni & 0,502 & 0,500 & 0,242 & & & & & \\
\hline 13 & ordinam. gerarchici & organizzażone gerarchica delle pagine di un sito & 0,491 & 0,500 & 0,363 & & & & & \\
\hline 8 & visual literacy & interpretażone di interfacce (barra di menu) & 0,487 & 0,500 & 0,231 & & & & & \\
\hline 42 & processi & esecuzione di un percorso guidato & 0,480 & 0,500 & 0,286 & & & & & \\
\hline 22 & sicurezza personale & dare fiducia a interlocutori occasionali in rete & 0,476 & 0,411 & 0,308 & & & & & \\
\hline 14 & ordinam. gerarchici & i organizzazione gerarchica di informazioni & 0,473 & 0,499 & 0,352 & & & & & \\
\hline 39 & comprens. tabella & soluzione di un problema sulla base di dati letti in tabella & 0,447 & 0,497 & 0,527 & & & & & \\
\hline 15 & comprens. tabella & soluzione di un problema sulla base di dati letti in tabella & 0,392 & 0,488 & 0,462 & & & & & \\
\hline 34 & digital reading & scelta di tag per un testo & 0,337 & 0,473 & 0,198 & & & & & \\
\hline 10 & digital reading & scelta di tag per un testo & 0,304 & 0,460 & 0,407 & & & & & \\
\hline
\end{tabular}

Figura 7. - Indici derivanti dalla item analysis. 
Con tutte le cautele necessarie (per la mancanza di affidabili riferimenti esterni e per la variabilità locale degli item ${ }^{9}$ ), ma comunque tenendo conto dei livelli di conoscenza e di competenza previsti per una classe quarta primaria, lo schema di Figura 7 ci consente di avanzare alcune riflessioni.

La dimensione risultata più difficile è quella cognitiva: dei dodici item appartenenti all'ultimo quartile ben nove riguardano abilità cognitive ${ }^{10}$. Solo un terzo dei bambini sa rintracciare le parole chiave di un testo, soltanto il $40 \%$ riesce a leggere una tabella operando delle scelte che soddisfino contemporaneamente due condizioni, meno del $50 \%$ riesce a fare una classificazione gerarchica o è in grado di scegliere la rappresentazione simbolica corretta di un processo dopo una esemplificazione della simbologia o di disegnare un percorso ottimale in un sistema righe/colonne.

All'opposto i bambini mostrano di saper affrontare le criticità tecnologiche più comuni (la stampa o l'audio che non funzionano, il programma che non si avvia, la pagina web che non viene visualizzata correttamente). Se la cavano meno bene quando devono valutare che cosa un computer possa o non possa fare (si veda l'esempio dell'avatar in Figura 4 e item analoghi): solo la metà risponde correttamente.

Sul piano etico e della sicurezza si rileva che oltre il $70 \%$ degli studenti della scuola primaria sa che le informazioni in rete possono non essere vere e che occorre dubitare, che non si devono inserire video o immagini senza il permesso dei diretti interessati, ma non sempre sanno come comportarsi in caso di phishing (item 22 e 46).

Le nette differenze tra una dimensione e l'altra, che emergono attraverso item ritenuti da molteplici valutatori adeguati all'età, ci inducono a raccomandare massima attenzione a quei processi cognitivi di alto livello che, pur essendo parte indispensabile della competenza digitale, sono anche trasversali alle altre literacy di base (competenza linguistica e numerica). Questo andamento risulta in linea con quanto già rilevato anche per adolescenti (Calvani, Fini, \& Ranieri, 2010) e quanto emerso da altri test internazionali (TIMSS, PIRLS, PISA).

9 Come noto la particolare formulazione dell'item e il grado di difficoltà generato dalle alternative di risposta può avere un ruolo decisivo. Ad esempio i quattro item identificati come «browsing - ricerca di informazioni in rete» ottengono punteggi difformi, due intorno al valore mediano e due nei quartili più alti, cioè più facili. Si tratta di item di pertinenza dell'informazione reperita in cui si simula una query in rete, si presentano le risposte fornite dal motore di ricerca e si chiede all'alunno di selezionare quelle pertinenti all'oggetto della ricerca. È evidente che le situazioni presentano gradi di difficoltà diversa in funzione delle diverse formulazioni dell' item e delle possibili risposte.

10 Occorre considerare anche che gli item cognitivi risultati più facili sono quelli legati al browsing, tra i più sottoposti a fattori di variabilità interna (si veda la nota precedente). 


\section{CONSIDERAZIONi DidATTICHE}

Le prove iDCA consentono una rapida ricognizione degli ambiti e dei gradi di intervento necessari da compiere con bambini della scuola primaria in materia di competenza digitale intesa secondo un modello che va oltre l'idea di una pura alfabetizzazione informatica.

La strutturazione del test in una versione unica ma anche in due prove equivalenti (ingresso e uscita) serve per orientare gli insegnanti a elaborare interventi volti a conseguire miglioramenti nei vari ambiti costitutivi della competenza digitale, consentendo loro di poterne valutare l'efficacia.

Numerose scuole che hanno applicato il questionario si sono fatte carico di allestire unità di intervento conseguenti, anche se non è stato possibile raccogliere i dati in modo sistematico a questo riguardo ${ }^{11}$. Per quanto ci è stato dato di vedere, l'efficacia dei risultati, normalmente constatabile anche con interventi di durata inferiore ad una decina di ore, oscilla a seconda del fatto che l'insegnante sappia ben ottimizzare il tempo disponibile con esercizi adeguatamente mirati agli obiettivi definiti dal test, senza invece disperdersi in attività di minore rilevanza; talvolta può essere sufficiente colmare semplici lacune conoscitive, come nel caso di conoscenze tecnologiche (funzionamento di un personal computer, comunicazioni in rete, messaggi di malfunzionamento, etc.).

Anche i quesiti in ambito etico spesso sono della stessa natura, fatto salvo che la loro effettiva messa in pratica rimane al di fuori di una possibile valutazione. Ad esempio, abbiamo verificato che molti bambini non sono a conoscenza dei rischi connessi all'adescamento o all'esistenza di truffe informatiche, tuttavia una volta mostrate loro adeguate esemplificazioni è facile renderli consapevoli del problema e dunque conseguire un sensibile incremento di risultato nel test d'uscita (altra cosa sarà vedere se poi si comporteranno in modo del tutto coerente con tale acquisizione).

Sono diverse invece le situazioni legate a problem solving, che chiamano in causa abilità cognitive più alte, di natura linguistica o logica. In questi casi la competenza digitale si correla con dimensioni più generali dell'intelligenza il cui sviluppo richiede tempi più lunghi ed un intervento integrato che può coinvolgere anche diverse discipline. E importante richiamare le difficoltà che i preadolescenti incontrano su questo aspetto, in contrasto con la mitologia diffusa dei «nativi digitali», secondo cui la semplice frequentazione tecnologica si accompagnerebbe anche allo sviluppo automatico di skill cognitive di particolare rilevanza e qualità. Una ipotesi schematica di tale dimensionamento temporale, in rapporto alle caratteristiche del Syllabus, è riportata in Figura 8.

11 In particolare molti insegnanti, partendo dalle prove iDCA, si sono mossi all'interno di una rete di esperienze promossa da Patrizia Appari (http://www.laboratorioformazione.it). 


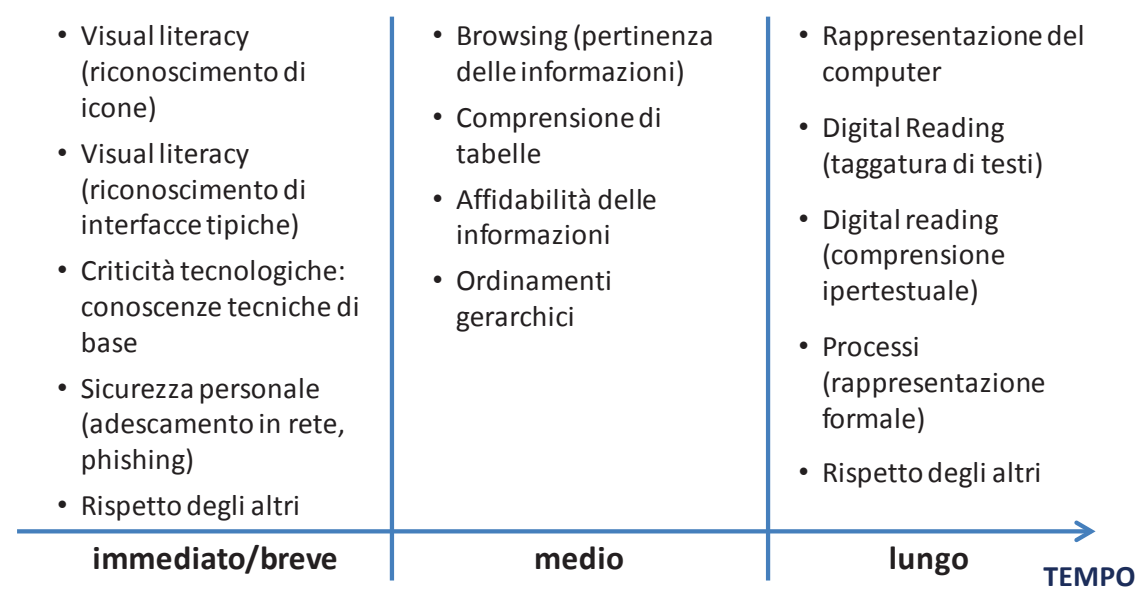

Figura 8. - Proposta di durata degli interventi didattici relativi alle componenti del Syllabus.

\section{Conclusioni}

In un recente lavoro uno dei più noti neuroscienziati che si avvale dei moderni sistemi di neuroimaging per studiare come il cervello si comporti dinanzi a compiti che comportano l'impiego di device tecnologici, ha gettato un grido d'allarme mostrando come i circuiti neuronali dei nativi digitali si stiano modificando per il sovraccarico derivante dall'intensa frequentazione con i media che inizia sin dai primissimi anni di vita: in sintesi si disattivano i circuiti legati alla capacità di attenzione, di rapporto interpersonale, in particolare per ciò che riguarda la comprensione delle emozioni dell'altro dall'osservazione del suo volto (Small \& Vorgan, 2008).

Chi cavalca l'onda dei nativi digitali è portato ingenuamente a perseguire l'ipotesi di adeguamento della scuola ai nuovi comportamenti giovanili, sulla base del fatto che le nuove tecnologie sono ormai un milieu da cui è impossibile prescindere. All'opposto, a fronte delle pratiche tecnologiche che spontanemente si diffondono nei giovani la scuola ha un compito di grande rilievo, vale a dire quello di favorire la migrazione all'interno dei nuovi media delle funzioni cognitive "alte» (lettura in profondità, selezione critica dell'informazione, chiarezza e coerenza logico-deduttiva) tipiche dei vecchi media. A tutto ciò vanno aggiunte altre istanze, quelle cioè di educare all'autocontrollo, alla consapevolezza etica dei diritti e doveri a cui ci si deve 
attenere nel mondo delle comunicazioni mediate da tecnologia. Non si tratta dunque di insegnare ai giovani ad usare le tecnologie nel senso di una banale alfabetizzazione tecnica - a questo livello possono cavarsela da sé con la pura frequentazione - ma ad usarle secondo modalità critiche ed eticamente consapevoli attivando quei circuiti nervosi superiori che la frequentazione spontanea tende piuttosto a disattivare.

Il luogo in cui si gioca questa partita si chiama oggi "competenza digitale» e per introdurre la sua formazione nella scuola sono intervenute raccomandazioni europee e politiche nazionali e internazionali.

In questo lavoro, nel quadro di un progetto di ricerca di strumenti di valutazione della competenza digitale ai diversi livelli di età (DCA), abbiamo presentato uno strumento che identifica le componenti essenziali di competenza digitale traducibile per bambini di scuola primaria, includendo anche abilità cognitive alte trasversali ai vari domini disciplinari.

L'applicazione su un campione di bambini della quarta primaria evidenzia criticità riportabili di fatto a due tipologie, quelle risolvibili con interventi brevi e con una buona informazione mirata (conoscenze specifiche sulle tecnologie o sull'etica della rete), quelle da rendere oggetto di interventi più sistematici e ricorsivi, che rimandano a criticità più generali, evidenziate anche da altri strumenti (TIMSS, PISA, PIRLS).

Da questo punto di vista il suggerimento che emerge si sposta all'impianto curricolare complessivo e alla necessità che processi cognitivi di buon livello, quali astrazione, ordinamento, sistemi di classificazione, gerarchizzazione di dati, rappresentazione formalizzata di processi, sintesi di un testo, lettura di una tabella e di un diagramma, siano messi sistematicamente al centro delle diverse attività svolte dagli educatori, muovendo dall'interno dei vari domini disciplinari.

\section{RIFERIMENTI BIBLIOGRAFICI}

ACRL (2000). Information literacy competency standards for higher education. Chicago: American Library Association.

ALA - American Library Association. Presidential Committee on Information Literacy (1989). Final report. http://www.ala.org/acrl/publications/whitepapers/ presidential (consulted 11/11/2014).

Ala-Mutka, K. (2011). Mapping digital competence: Towards a conceptual understanding. JRC technical notes, JRC67075, Seville: Institute for Prospective Technological Studies. http://ftp.jrc.es/EURdoc/JRC67075_TN.pdf (consulted $11 / 11 / 2014)$. 
Bruce, B., \& Peyton, J. K. (1999). Literacy development in network-based classrooms: Innovation and realizations. International Journal of Educational Technology, 1(2), 1-27.

Calvani, A., Fini, A., Molino, M., \& Ranieri, M. (2009a). Visualizing and monitoring effective interactions in online collaborative groups. British Journal of Educational Technology, 2, 1-14.

Calvani, A., Fini, A., \& Ranieri, M. (2009b). Assessing digital competence in secondary education - Issues, models, instruments. In M. Leaning (Ed.), Issues in information and media literacy. Education, practice and pedagogy, Santa Monica, CA: ISI.

Calvani, A., Fini, A., \& Ranieri, M. (2009c). Valutare la competenza digitale. Modelli teorici e strumenti applicativi. TD Tecnologie Didattiche, 17(3), 39-46. http://www.tdjournal.itd.cnr.it/files/pdfarticles/PDF48/6_Calvani_ Fini_Ranieri_TD48.pdf (consulted 11/11/2014).

Calvani, A., Fini, A., \& Ranieri, M. (2010). La competenza digitale nella scuola. Modelli e strumenti per valutarla e svilupparla. Trento: Erickson.

Calvani, A., Fini, A., Ranieri, M., \& Picci, P. (2012). Are young generations in secondary school digitally competent? A study on Italian teenagers. Computers \& Education, 58(2), 797-807. http://www.uio.no/studier/emner/matnat/ifi/INF3280/ v13/undervisningsmateriale/calvanietal2012digitalcompetenceitalianteenagers. pdf (consulted 11/11/2014).

Calvani, A., \& Menichetti, L. (2013). La competenza digitale: per un modello pedagogicamente significativo. TD Tecnologie Didattiche, 21(3), 132-140. http:// www.tdjournal.itd.cnr.it/files/pdfarticles/PDF60/TD61_0_competenza.pdf (consulted 11/11/2014).

Crandall, B., Klein, G., \& Hoffman, R. R. (2006). Working minds: A practitioner's guide to cognitive task analysis. Cambridge, MA: MIT Press.

Davies, J., Szabo, M., \& Montgomerie, C. (2002). Assessing information and communication technology literacy of education undergraduates: Instrument development. In P. Barker \& S. Rebelsky (Eds.), Proceedings of EDMEDIA 2001 World Conference on educational multimedia, hypermedia and telecommunications (pp. 377-383). Denver - Norfolk, VA: Association for the Advancement of Computing in Education.

Didoni, R., Quattrocchi, S., Menichetti, L., \& Calvani, A. (2013). Valutare la competenza digitale come competenza complessa nella scuola secondaria. TD Tecnologie Didattiche, 21(1), 30-34. http://www.tdjournal.itd.cnr.it/files/ pdfarticles/PDF58/td58_5_didoni.pdf (consulted 11/11/2014).

DigComp (2010-2012). http://is.jrc.ec.europa.eu/pages/EAP/DIGCOMP.html (consulted 11/11/2014).

Eshet-Alkalai, Y. (2004). Digital literacy: A conceptual framework for survival skills in the digital era. Journal of Educational Multimedia and Hypermedia, 13(1), 93-106. 
EU (2006). Recommendation of the European Parliament and of the Council of 18 December 2006 on key competences for lifelong learning (2006/962/ EC). Official Journal of the European Union, L 394, 10-18.

Ferrari, A. (2012). Digital competence in practice: An analysis of frameworks. Sevilla: Institute for Prospective Technological Studies. http://ftp.jrc.es/EURdoc/ JRC68116.pdf (consulted 11/11/2014).

Hattie, J. (2009). Visible learning. A Synthesis of over 800 meta-analyses relating to achievement. London - New York: Routledge.

Janssen, J., \& Stoyanov, S. (2012). Online consultation on experts' views on digital competence. http://ftp.jrc.es/EURdoc/JRC73694.pdf (consulted 11/11/2014).

Li, Y., \& Ranieri, M. (2010). Are «digital natives» really digitally competent? A study on chinese teenagers. British Journal of Educational Technology. doi: 10.1111/j.1467-8535.2009.01053.x.

NAEP (2010). Technology and engineering literacy assessment and item specifications for the 2014 National Assessment of Educational Progress. WestEd. http://www.edgateway.net/cs/naepsci/view/naep_nav/9 (consulted $11 / 11 / 2014)$.

OECD (2011). PISA 2009 results. Students on line: Digital technologies and performance, Vol. VI. Paris: OECD.

Olimpo, G. (2013). Riflessioni brevi su digital literacy e digital competence. TD Tecnologie Didattiche, 21(1), 14-18. http://www.tdjournal.itd.cnr.it/files/ pdfarticles/PDF58/td58_2_olimpo.pdf (consulted 11/11/2014).

Prensky, M. (2001). Digital natives, digital immigrant. II Part: Do they really think differently? On the Horizon, 9(6), 15-24.

Silva, E. (2008). Misurare le competenze per il XXI secolo. ADI, Associazione Docenti Italiani. http://www.adiscuola.it (consulted 11/11/2014).

Small, G., \& Vorgan, G. (2008). iBrain: Surviving the technological alteration of the modern mind. Bloomington, IN: Collins Living.

Swan, K., Bangert-Drowns, J. B., Moore-Cox, A., \& Dugan, R. (2002). Technology $\&$ literacy learning: A national survey of classroom use. In P. Barker, S. Rebelsky (Eds.), Proceedings of ED-MEDIA 2001 World Conference on educational multimedia, hypermedia and telecommunications (pp. 221-223). Denver Norfolk, VA: Association for the Advancement of Computing in Education.

Wiggins, G. P. (1991). A true test: Towards more authentic and equitable assessment. Phi Delta Kappan, 70(9), 703-713. 


\section{RiassunTO}

La competenza digitale è ormai riconosciuta come una delle finalità educative più rilevanti da perseguire nelle politiche del nuovo millennio. Numerose sono le riflessioni che la ricerca sta avanzando per definirne meglio la natura, generalmente convergenti nel sottolineare la necessità di concepire modelli teorici nei quali, accanto a conoscenze e abilità strettamente tecniche, siano presenti abilità cognitive e nozioni sia etiche che comportamentali. In accordo con la riflessione teorica si pone il problema di fornire alle scuole strumenti per favorire e valutare i progressivi avanzamenti ai vari livelli di età. Nel quadro del "frame» europeo e all'interno di un programma che concerne anche altri livelli scolari (DCA Digital Competence Assessment) nel presente contributo viene illustrato un questionario di valutazione della competenza digitale utilizzabile nella scuola primaria, utile anche per individuare ambiti sui quali effettuare interventi immediati. Il questionario, corredato delle relative istruzioni d'uso, è reso disponibile in rete in modalità Creative Commons.

Parole chiave: Competenze digitali, Modello, Questionario, Scuola primaria, Valutazione. 